Europhysics Letters

PREPRINT

\title{
Overcharging: The crucial role of excluded volume
}

\author{
R. Messina ${ }^{1}(*)$, E. González-Tovar ${ }^{2,3,4}(* *)$, M. Lozada-Cassou ${ }^{2,4}\left({ }^{* * *}\right)$ and C. \\ $\operatorname{HoLM}^{1}(\stackrel{* *}{*})$ \\ 1 Max-Planck-Institut für Polymerforschung - Ackermannweg 10, 55128 Mainz, Ger- \\ many \\ 2 Departamento de Fúsica, Universidad Autónoma Metropolitana-Iztapalapa - Apartado \\ Postal 55-534, 09340 D.F., México \\ 3 Instituto de Física, Universidad Autónoma de San Luis Potosí - Álvara Obregón 64, \\ 78000 San Luis Potosí, México \\ 4 Programa de Ingeniería Molecular, Instituto Mexicano del Petróleo - Lázaro Cardenas \\ 152, 07730 D. F., México
}

PACS. 61.20.Qg - Structure of associated liquids: electrolytes, molten salts, etc..

PACS. 82.70.Dd - Colloids.

PACS. 87.15.Aa - Theory and modeling; computer simulation.

\begin{abstract}
In this Letter we investigate the mechanism for overcharging of a single spherical colloid in the presence of aqueous salts within the framework of the primitive model by molecular dynamics (MD) simulations as well as integral-equation theory. We find that the occurrence and strength of overcharging strongly depends on the salt-ion size, and the available volume in the fluid. To understand the role of the excluded volume of the microions, we first consider an uncharged system. For a fixed bulk concentration we find that upon increasing the fluid particle size one strongly increases the local concentration nearby the colloidal surface and that the particles become laterally ordered. For a charged system the first surface layer is built up predominantly by strongly correlated counterions. We argue that this a key mechanism to produce overcharging with a low electrostatic coupling, and as a more practical consequence, to account for charge inversion with monovalent aqueous salt ions.
\end{abstract}

Overcharging, or charge inversion, is defined as the situation where a charged colloid (macroion) accumulates close to its surface more counterions than necessary to compensate its own bare charge. This effect was already discovered in the beginning of 80's, both by computer simulations [1] and analytical studies [2,3]. Based on reversed electrophoretic mobility, some experimental [4] and numerical (MD) [5] studies provide some hints for the manifestation of overcharging and its possible experimental relevance. More recently, it has regained a considerable attention on the theoretical side [6] 16].

(*) E-mail: messina@mpip-mainz.mpg.de

(**) E-mail: henry@dec1.ifisica.uaslp.mx

(***) E-mail: marcelo@www.imp.mx

$\left(*^{*}\right)$ E-mail: holm@mpip-mainz.mpg.de

(C) EDP Sciences 
Overcharging is rather well understood for counterions in salt-free or low salt environment, when excluded volume effects play no role. The underlying physics at zero temperature of such non-neutral systems can be quantitatively explained with Wigner crystal (WC) theory [6 8, 11]. The basic concept is that the counterions form a two-dimensional lattice on the macroion surface, and when overcharging counterions are present on this layer the energy of the system is lowered compared to the neutral case. This feature can be directly and exactly computed for a small number of counterions at zero temperature, and was illustrated in Refs. [11]. This WC approach remains qualitatively correct for finite temperature as long as the Coulomb coupling is very high. The crystal then melts into a strongly correlated liquid, where the local order is still strong enough to lower the free energy for the overcharged state.

The situation becomes much more complicated for aqueous systems, where the coupling is weak and in addition salt is present. One-particle inhomogeneous integral equation theories can describe some of these situations fairly well [2, 3, 14, 16] but the computed correlation functions do not necessarily give direct insight into the physical mechanisms behind these effects. The presence of excluded volume interactions can lead to layering effects near the macroion, which are known from simple fluid theories. Here, due to the presence of charge carriers of both signs, this can even lead to layers of oscillating charge inversions [3, 14, 17]. This overcharging was also observed (by integral-equation and simulation [3, 15]) for monovalent salt-ions of large size. However, until today, the basic mechanism of charge inversion for dense salt solutions remains unclear.

The goal of this Letter is (i) to study in detail the role of the excluded volume contribution for the overcharging of a colloidal macroion in the presence of salts and (ii) to provide a qualitative insight into the mechanism behind these effects. We find that in the presence of salt the contribution of excluded volume can be so important that the size of the small ions dominates the occurrence of overcharging and the overcharging strength increases with increasing ionic size when the electrolyte concentration is fixed. Even for monovalent ions with high enough ionic size, we observe overcharging, which can not be explained with a salt-free WC picture due to the low Coulomb coupling strength. In order to have the simplest system we study only the cases where the coion and the counterions have the same size. This will reduce the effects of depletion forces which lead to nontrivial features already in neutral hard sphere fluids.

Our proposed mechanism will rest on the following arguments: For a fixed salt concentration, the available volume in the fluid is function of the electrolyte particle size. More precisely, the entropy of the solution is decreased by enlarging the size of the salt-ions [18], which enhances inter-particle correlations. On the other hand the interface provided by the macroion leads to an increase of microion density close to the macroion, and promotes there also lateral ordering, even in the absence of strong electrostatic coupling, similar to a prefreezing phenomenon. However entropy alone can never lead to overcharging, since in this limiting case coions and counterions have the same radial distribution. But ordering and weak electrostatic correlations can lead to overcharging, as we are going to prove in this Letter.

In this work we carried out MD simulations and HNC/MSA (hypernetted chain/mean spherical approximation) integral-equation to study the overcharging in spherical colloidal systems within the primitive model. In particular, the solvent enters the model only by its dielectric constant and its discrete structure 19 is ignored. The system is made up of (i) a large macroion with a bare central charge $Q=-Z_{m} e$ (with $Z_{m}>0$ ) and (ii) symmetric salt ions of diameter $\sigma$ and valence $Z_{c}$. The system is globally electrically neutral.

For the simulation procedure, all these ions are confined in an impermeable cell of radius $R$ and the macroion is held fixed at the cell center. In order to simulate a canonical ensemble, the Langevin thermostat has been used to predict the ions trajectory as has been done in 
TABLE I - Simulation run parameters for the charged fluid $(A-F)$ and the neutral fluid $(G-H)$.

\begin{tabular}{ccccc}
\hline parameters & salt valence & $Z_{m}$ & $\sigma / l_{B}$ & $f$ \\
\hline $\operatorname{run} A$ & $2: 2$ & 48 & 1 & $2.3 \times 10^{-1}$ \\
$\operatorname{run} B$ & $2: 2$ & 48 & 0.5 & $2.9 \times 10^{-2}$ \\
$\operatorname{run} C$ & $2: 2$ & 48 & 0.25 & $3.6 \times 10^{-3}$ \\
$\operatorname{run} D$ & $1: 1$ & 10 & 1 & $2.3 \times 10^{-1}$ \\
$\operatorname{run} E$ & $1: 1$ & 48 & 1 & $2.3 \times 10^{-1}$ \\
$\operatorname{run} F$ & $1: 1$ & 48 & 0.5 & $2.9 \times 10^{-2}$ \\
$\operatorname{run} G$ & - & - & 1 & $2.3 \times 10^{-1}$ \\
$\operatorname{run} H$ & - & - & 0.5 & $2.9 \times 10^{-2}$ \\
\hline
\end{tabular}

Refs. [1].

Excluded volume interactions are taken into account with a purely repulsive (6-12) LennardJones (LJ) potential characterized by the standard $(\sigma, \epsilon)$ length and energy parameters, respectively, which is cut-off at the minimum [20]. The macroion-counterion distance of closest approach is defined as $a$. Energy units in our simulations are fixed by $\epsilon=k_{B} T$ (with $T=298$ $\mathrm{K})$.

The pair electrostatic interaction between any pair $i j$, where $i$ and $j$ denote either a macroion or a microion, reads $U_{\text {coul }}(r)=k_{B} T l_{B} \frac{Z_{i} Z_{j}}{r}$, where $l_{B}=e^{2} /\left(4 \pi \epsilon_{0} \epsilon_{r} k_{B} T_{0}\right)$ is the Bjerrum length fixing the length unit. Being interested in aqueous solutions we choose the relative permittivity $\epsilon_{r}=78.5$, corresponding to $l_{B}=7.14 \AA$. The time step is $\Delta t=0.01 \tau$ with $\tau=\Gamma^{-1}$, where $\Gamma$ is the damping constant of the thermostat. Typical simulation runs consisted of $2-7 \times 10^{6} \mathrm{MD}$ steps after equilibration.

In order to give prominence to the effect of ionic size, we choose to work at fixed distance of closest approach (between the centers of macroion and the salt-ion) $a=2 l_{B}=14.28 \AA$. In this way, the electrostatic correlation induced by the colloid remains the same (i.e. fixed macroion electric field at contact) no matter what the ionic size is. Thus by changing the ionic size $\sigma$ one also changes that colloidal radius accordingly so that $a$ remains constant. The salt concentration $\rho$ is given by $N_{-} / V$ where $V=\frac{4}{3} \pi R^{3}$ is the cell volume and $N_{-}$is the number of coions. We will restrict the present study to $\rho=1 \mathrm{M}$ salt concentration. The fluid volume fraction $f$ is defined as $\left(N_{+}+N_{-}\right)\left(\frac{\sigma}{2 R}\right)^{3}$ where $N_{+}$is the number of counterions. To avoid size effect induced by the simulation cell we choose a sufficiently large radius $R=8.2 l_{B}$ yielding to more than 1000 mobile charges. Simulation run parameters are gathered in Table $\mathbb{1}$. The HNC/MSA calculations were performed using the technique presented in [16] and references therein. Here it is assumed that the system size is infinite, and the bulk salt concentration is fixed. In practice there should be no observable difference in the correlation functions (between HNC/MSA and MD) close to the macroions, because the wall effects die off sufficiently fast.

We first illustrate the excluded volume correlations present for a neutral hard sphere fluid (runs $G-H$ ) [identical to systems $A-B$ and $E-F$, but uncharged (see Table $\mathbb{1}$ )]. Although these kind of systems are "simple" fluids [21], it is fruitful to elucidate what exactly happens at this "low" fluid density upon varying the fluid particle size $\sigma$ in the presence of a single large spherical particle. To characterize the fluid structure we consider the pair distribution function $g(r)$ between the colloid and the fluid particle, which is just proportional to the local density $n(r): n(r)=\rho g(r)$. Results are depicted in Fig. (11). For the large value $\sigma=l_{B}$, one clearly observes a relative high local concentration as well as a short range ordering nearby the colloidal surface. When the particle size is reduced by a factor 2 (holding $a$ fixed), the behavior is qualitatively different and the system is basically uncorrelated. By increasing $\sigma$ at fixed fluid density $\rho$ the mean surface-surface distance between particles is reduced which 


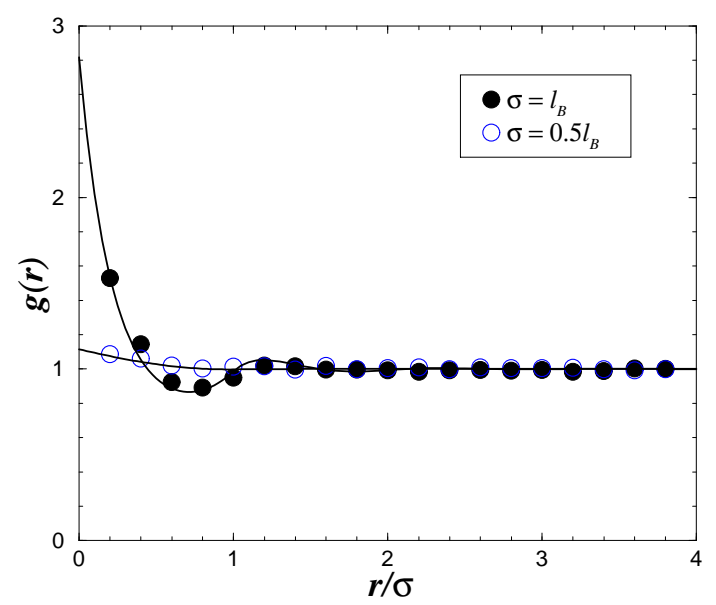

Fig. 1 - Pair distribution function $g(r)$ between the colloid and the fluid particle in the neutral state (runs $G-H$ ) for two particle sizes $\sigma$. The origin of the abscissa is taken at the distance of closest approach $a=2 l_{B}$. Lines and symbols correspond to HNC theory and simulation respectively.

in turn leads to higher collision probability and thus to higher correlations. In other words, by reducing the available volume one promotes ordering [16, 22]. This seems to be trivial in the bulk, but the presence of the colloidal surface induces an even enhanced ordering and the system can prefreeze (order) close to the colloid. Note that the same effects are naturally present for a fluid close to a planar wall [21,23].

To characterize the overcharging we introduce the fluid integrated charge $Q(r)$ which corresponds to the total net charge in the fluid (omitting the macroion bare charge $Z_{m}$ ) within a distance $r$ from the distance of closest approach $a$. Results are sketched in Fig. 2(a) and Fig. 2(b) for divalent and monovalent salt ions respectively. Theoretical and numerical analysis are in very good qualitative agreement.

For the divalent electrolyte solutions (runs $A-C$ ) we observe that overcharging is strongly dictated by the ionic size $\sigma$ [see Fig. 2(a)]. For small ions (run $C$ ) no overcharging occurs (i. e. $Q(r) / Z_{m}<1$ ), which is a non trivial effect probably related to the forming of ionic pairs (counterion-coion pairing) due to the strong pair interaction of $8 k_{B} T$. This delicate point will be addressed in a future study, and was also observed in [15]. Upon increasing $\sigma$, the degree of overcharging increases. We carefully checked that the distance $r=r^{*}$, where $Q\left(r^{*}\right)$ assumes its maximal value, corresponds to a zone of coion depletion (in average there are less than 2 coions within $r^{*}$ ). This implies that also the absolute number of counterions at the vicinity of the macroion surface increases with increasing $\sigma$. This is qualitatively in agreement with what we observed above for neutral systems. However we are going to show later that electrostatic correlations are also concomitantly responsible of this extra counterion population (increasing with $\sigma$ ) in the vicinity of the colloidal surface.

For the monovalent electrolyte solution [see Fig. 2(b)] overcharging occurs for $\sigma=l_{B}$ (runs $D$ and $E$ ). In respect to the salt-free WC picture this is rather unexpected since here the "plasma" parameter $\Gamma_{c c}=l_{B} Z_{c}^{2} / a_{c c}$, where $a_{c c}=(\pi c)^{-1 / 2}$ (c standing for the twodimensional surface counterion concentration) is the mean distance between counterions on the surface, is small. More precisely for $Z_{m}=10$ (run $D$ ) we find $\Gamma_{c c} \approx 0.8$, and for $Z_{m}=48$ (run $E$ ) we have $\Gamma_{c c}<1.0$ [24]. But following this salt-free approach, it is necessary to have 

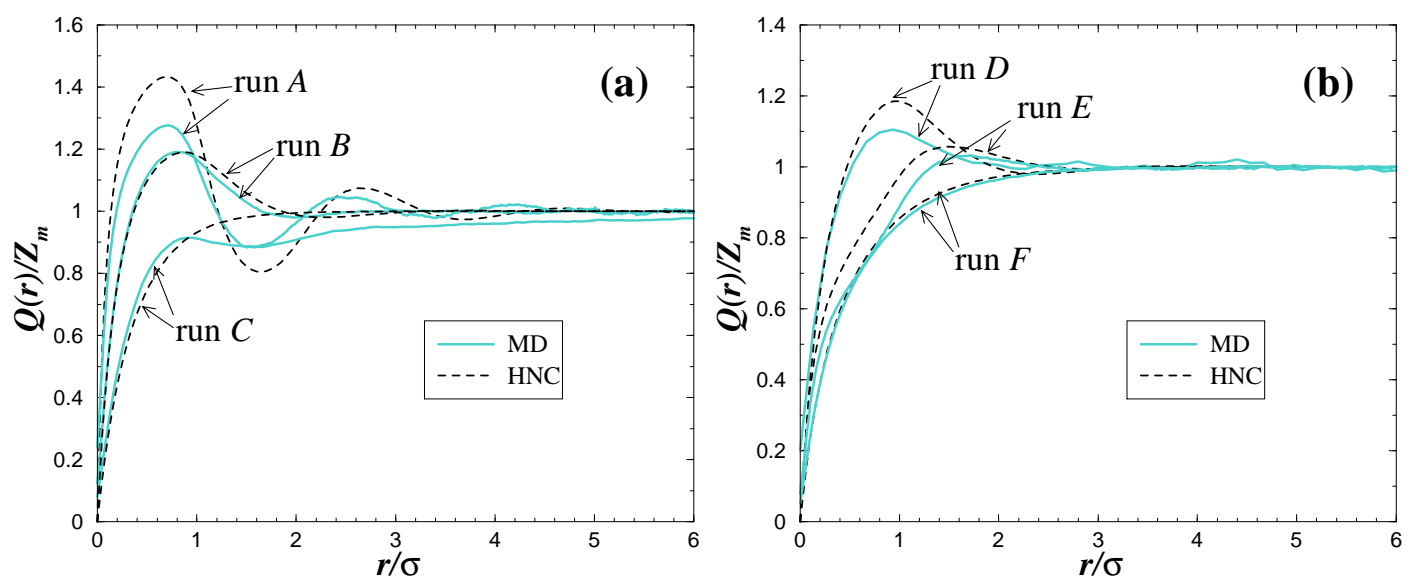

Fig. 2 - Reduced fluid integrated charge $Q(r) / Z_{m}$ as a function of distance $r$ for for three different particle sizes $\sigma$. (a) divalent salt ions (runs $A-C$ ), (b) monovalent salt ions (runs $D-F$ ). The origin of the abscissa is taken at the distance of closest approach $a=2 l_{B}$

at least $\Gamma_{c c}>2$ to get overcharge [25]. Note that from run $D\left(Z_{m}=10\right)$ to run $E\left(Z_{m}=48\right)$ one increases the macroion surface-charge density leading (for $Z_{m}=48$ ) to a higher absolute overcharging $Q\left(r^{*}\right)$ but a weaker ratio $Q\left(r^{*}\right) / Z_{m}$, which is qualitatively in accord with the WC picture, since the maximal overcharging is proportional to $\sqrt{c}$. A closer look on Fig. 2(b) reveals that for $Z_{m}=48$ (run $E$ ) $r^{*} / \sigma$ is shifted to the right compared to the divalent case. This is merely a packing effect and it is due to the fact that for monovalent counterions the macroion charge (over)compensation involves twice more number of counterions particles than in the divalent case. Therefore for the macroion charge density under consideration $\left(Z_{m}=48\right)$, more than one counterion-layer is needed to compensate the macroion charge. Again for a smaller ionic salt size $\sigma=0.5 l_{B}$ ( run $F$ ) the overcharging effect is canceled.

Recently a depletion of salt-ions (total local density of co and counterions) near the macroion surface was reported for bulk salt-ion concentrations similar to ours but very low surface charge density [26]. For all of our investigated cases (runs $A-F$ ), however, we never observe such a depletion. The reason is that such an effect vanishes as soon as the ionic size is sufficiently large and/or the surface-charge density is large enough, which is in agreement with our findings. Indeed, similarly to what happened with image charges, the self-image repulsion is only relevant for low charge density [27]. Note that the parameters leading to overcharging cannot give rise to an observation of salt-ion depletion.

For the WC picture to be effective we need strong lateral correlations which cannot come from pure electrostatic effects. To see if such correlations are present we consider the local twodimensional surface counterion structure. We analyzed in our simulations the two-dimensional counterion pair distribution $g_{c c}(s)$, where $s$ is the arc length on the macroion sphere of radius $a$. All counterions lying at a distance $r<a+0.5 \sigma$ from the macroion center are radially projected to the contact sphere of radius $a$. Predominantly counterions are present in the first layer. For the neutral system ( run $G$ ) we analyzed the small neutral species. Results are given in Fig. 3. We observe that all systems with an ion diameter $\sigma=l_{B}$ show their first peak at roughly $1 \sigma$, and a weaker second peak at about $2 \sigma$, exhibiting long range surface correlations. The second peak is very weak in the neutral system $G$, but clearly visible in system $A$ and $E$. Due to the stronger electrostatic repulsion the second peak for the divalent 


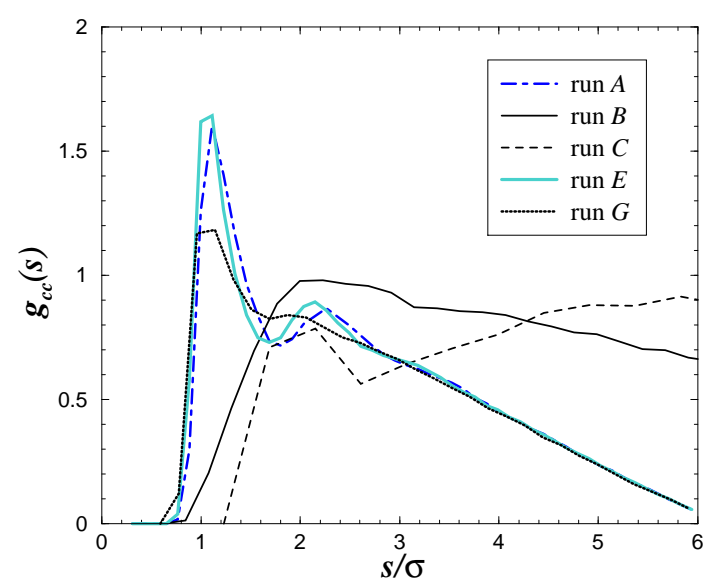

Fig. 3 - Two-dimensional surface counterion correlation functions $g_{c c}(s)$ for divalent salt (run $A-C$ ), monovalent salt (run $E$ ), and a neutral system (run $G$ ).

system $A$ is somewhat further apart than for the monovalent system $E$. The systems $B$ and $C$ with smaller ion diameters show a correlation hole of size $\approx 2 \sigma$, which is of purely electrostatic origin. Therefore lateral correlations can be produced either by pure entropy effects (run $G$ ) or pure electrostatic effects (run $C$ ), or we can have an enhanced lateral ordering due to the interplay of both (run $A, E$ ). Qualitatively, WC arguments are still applicable to monovalent systems (such as run $E$ ) if one considers an effective low local surface temperature stemming from the strong surface ordering.

In summary, the observed overcharging for low Coulomb coupling can be traced back to the complicated interplay of entropy and energy by the following two effects. First, by enlarging the excluded volume of the salt ions at fixed concentration, one lowers the accessible volume to the fluid particles. Second, the presence of the large macroion provides an interface near which the density of the fluid is increased compared to the bulk, and the solution can prefreeze due to entropic effects, provided the available volume gets low enough. The closest layer to the interface possesses already strong lateral correlations, even for a neutral system. If the system is additionally charged, then even weak Coulomb correlations can lead to the formation of a strongly correlated liquid, where the overcharged state is energetically favorable, as shown in [7, 11, 12, 16]. The order of this counterion layer is however not created by electrostatic interactions as in the normal WC picture, but it is largely due to entropic effects. The observed overcharging effect might have implications for the stability of colloidal suspensions. Additions of monovalent salt will eventually make colloidal suspensions unstable due to the onset of the van-der-Waals attractions. Upon addition of even more monovalent salt there is the possibility of salting the precipitate again in, as has been seen for polyelectrolyte systems [28]. The observation of such a reentrant transition could be an important hint towards the existence of overcharging with monovalent salt ions.

We are grateful to K. Kremer for a critical reading of the manuscript, and R. M. thanks the LEA for financial support. E. G.-T. acknowledges the support by PROMEP and FAI-UASLP. 


\section{REFERENCES}

[1] van Megen W. and Snook I., J. Chem. Phys., 73 (1980) 4656; Torrie G. M., and Valleau J. P., 73 (1980) 5807.

[2] Lozada-Cassou M., Saavedra-Barrera R. and Henderson H., J. Chem. Phys., 77 (1982) 5150.

[3] Gonzales-Tovar E., Lozada-Cassou M. and D. Henderson, J. Chem. Phys., 83 (1985) 361.

[4] Galisteo F., de las Nieves López F. J., Cabrerizo M. and Hidalgo-Alvarez R., Prog. Colloid Polym. Sci., 82 (1990) 313; Elimelech M. and O'Melia C. R., Colloids Surf., 44 (1990) 165; Walker H. W. and Grant S. B., Colloids Surf. A, 119 (1996) 229; Götting N., Fritz H., Maier M., von Stamm J., Schoofs T. and Bayer E., Colloid Polym. Sci., 277 (1999) 145.

[5] Tanaka M. and Grosberg A. Y., Eur. Phys. J. E, 7 (2002) 371.

[6] Shklovskit B. I., Phys. Rev. Lett., 82 (1999) 3268.

[7] Shklovskit B. I., Phys. Rev. E, 60 (1999) 5802.

[8] Nguyen T. T., Grosberg A. Y. and Shklovskit B. I., Phys. Rev. Lett., 85 (2000) 1568; J. Chem. Phys., 113 (2000) 1110.

[9] Lozada-Cassou M., González-Tovar E. and Olivares W., Phys. Rev. E, 60 (1999) R17.

[10] Nguyen T. T. and Shklovskit B. I., Phys. Rev. E, 64 (2001) 041407.

[11] Messina R., Holm C. and Kremer K., Phys. Rev. Lett., 85 (2000) 872; Europhys. Lett, 51 (2000) 461; Phys. Rev. E, 64 (2001) 021405.

[12] Messina R., Holm C. and Kremer K., Eur. Phys. J. E, 4 (2001) 363; Messina R., Physica $A, \mathbf{3 0 8}(2002) 59$.

[13] Tanaka M. and Grosberg A. Y., J. Chem. Phys., 115 (2001) 567.

[14] Greberg H. and KJellander R., J. Chem. Phys., 108 (1998) 2940.

[15] Deserno M., Jiménez-Ángeles F., Holm C. and Lozada-Cassou M., J. Phys. Chem. B, 105 (2001) 10983.

[16] Lozada-Cassou M. and Jiménez-Ángeles F., arXiv:physics/0105043 v2 (2001).

[17] Deserno M., May, S., and Holm C., Macromol., 33 (2000) 199.

[18] This argument holds far enough from the solid-liquid transition as in the present study (i. e. the highest fluid volume fraction $f$ we consider is 0.23 ). Indeed entropy alone can drive crystallization as in hard-sphere systems.

[19] Allahyarov E. And Löwen H., J. Phys. Condens. Matter, 13 (2001) L277; Phys. Rev. E, 63 (2001) 041403.

[20] The LJ potential is rigorously speaking not a hardcore one, but it can be considered in practice as such.

[21] Hansen J.-P. and McDonald I., Theory of Simple Liquids (Academic, London) 1990.

[22] This feature holds only if the fixed particle (here the colloid) is larger than the fluid particles.

[23] Courtemanche D. J. and van Swol F., Phys. Rev. Lett., 69 (1992) 2078; Götzelmann B., Haase A. and Dietrich S., Phys. Rev. E, 53 (1996) 3456; Kegel W. K., J. Chem. Phys., 115 (2001) 6538.

[24] This is due to a packing effect which imposes that $a_{c c}$ cannot be smaller than $\sigma$.

[25] Rouzina I. and Bloomfield V., J. Chem. Phys., 100 (1996) 9977.

[26] Allahyarov E., Löwen H., Louis A. A. and Hansen J. P., Europhys. Lett, 57 (2002) 731.

[27] Messina R., e-print:cond-mat/0204550, Image charges in spherical geometry: Application to colloidal systems.

[28] Eisenberg, H., Mohan, G.R., J. Phys. Chem., 63 (1959) 671; Eisenberg, H., Casassa, E.F., J. Polym. Sci., 47 (1960) 29; 